\title{
Binding language: structuring sentences through precisely timed oscillatory mechanisms
}

\author{
Katrien Segaert, ${ }^{1,2,3}$ (D) Ali Mazaheri ${ }^{1,2}$ and Peter Hagoort ${ }^{3,4}$ \\ ${ }^{1}$ School of Psychology, University of Birmingham, Edgbaston, Birmingham, UK \\ ${ }^{2}$ Centre for Human Brain Health, University of Birmingham, Birmingham, UK \\ ${ }^{3}$ Max Planck Institute for Psycholinguistics, Nijmegen, The Netherlands \\ ${ }^{4}$ Centre for Cognitive Neuroimaging, Donders Institute for Brain, Cognition and Behaviour, Radboud University Nijmegen, \\ Nijmegen, The Netherlands
}

Keywords: alpha and beta oscillations, EEG, language, syntactic binding

\begin{abstract}
Syntactic binding refers to combining words into larger structures. Using EEG, we investigated the neural processes involved in syntactic binding. Participants were auditorily presented two-word sentences (i.e. pronoun and pseudoverb such as 'I grush' and 'she grushes', for which syntactic binding can take place) and wordlists (i.e. two pseudoverbs such as 'pob grush' and 'pob grushes', for which no binding occurs). Comparing these two conditions, we targeted syntactic binding while minimising contributions of semantic binding and of other cognitive processes such as working memory. We found a converging pattern of results using two distinct analysis approaches: one approach using frequency bands as defined in previous literature, and one data-driven approach in which we looked at the entire range of frequencies between 3 and $30 \mathrm{~Hz}$ without the constraints of pre-defined frequency bands. In the syntactic binding (relative to the wordlist) condition, a power increase was observed in the alpha and beta frequency range shortly preceding the presentation of the target word that requires binding, which was maximal over frontal-central electrodes. Our interpretation is that these signatures reflect that language comprehenders expect the need for binding to occur. Following the presentation of the target word in a syntactic binding context (relative to the wordlist condition), an increase in alpha power maximal over a left-lateralised cluster of frontal-temporal electrodes was observed. We suggest that this alpha increase relates to syntactic binding taking place. Taken together, our findings suggest that increases in alpha and beta power are reflections of distinct the neural processes underlying syntactic binding.
\end{abstract}

\section{Introduction}

For language processing, it is common to make a distinction between two crucial components. The first component is memory for the linguistic properties of single words. This knowledge gets encoded and consolidated during language acquisition and is usually referred to as the mental lexicon. Language processing however entails a great deal more than retrieving single words from memory. Rather, the expressive power of human language is based on the ability to combine a limited set of individual words in novel ways to make up new sentences. The binding process that allows us to combine words into larger structures with new and complex meaning has been referred to as

Correspondence: Dr Katrien Segaert, as above.

E-mail: k.segaert@bham.ac.uk

Received 31 July 2017, revised 6 December 2017, accepted 14 December 2017

Edited by Dr. Heleen Slagter

Reviewed by Andrew Dimitrijevic, Sunnybrook Health Sciences Centre, Canada Lars Meyer, Max Planck Institute for Human Cognitive and Brain Sciences, Germany

The associated peer review process communications can be found in the online version of this article.
Merge (Chomsky, 1995; Grodzinsky \& Friederici, 2006; Zaccarella \& Friederici, 2015; Zaccarella et al., 2017) or Unification (Hagoort, 2005, 2009, 2016). This binding process is the second crucial component of language processing and is the essence of both language production and comprehension when we use language that goes beyond the single-word level. Binding therefore is a central topic of study in sentence processing research. The process of binding needs to happen at multiple levels. At the level of phonology, words are bound into intonational phrases. Another level of binding is semantic binding: the construction of complex meaning when words are combined into phrases and sentences. A third level is syntactic binding: the combination of words into larger structures, taking into account features that mark syntactic structure, tense, aspect and agreement. Syntactic binding is what we focus on in this study. Before introducing our study, we would like to highlight the progress that has been made in previous research on the topic of binding.

\section{The neurobiology of binding}

Binding at the most basic level would be the binding of two words in a minimal sentence or phrase. Such minimal binding lies at the 
core of more complex sentence comprehension and perhaps because it is so fundamental has attracted increasing research interest in recent years. Bemis \& Pylkkänen (2011) investigated the neural changes associated with nouns in a minimal binding context (e.g. red boat) and a wordlist condition (e.g. cup and boat) in an MEG study. Binding for a two-word phrase such as 'red boat' involves semantic as well as syntactic binding. For the wordlist condition (i.e. a list of unrelated words), retrieval of lexico-semantic information from memory takes place, but no binding occurs as no phrasal structure can be built for the words in the list. 'Red boat' and 'cup, boat' thus differ only in the presence vs. absence of the binding process: the construction of a syntactic and a semantic relationship. In Bemis \& Pylkkänen (2011), wordlists and phrases were presented visually and binding was associated with an evoked response in frontal areas (ventro-medial prefrontal cortex - vmPFC), following an evoked response in temporal areas (left anterior temporal lobe 1ATL).

The binding process that takes place when we process a linguistic expression such as a two-word sentence or phrase is the foundation for processing more complex sentences. The nature of the binding process is invariant. Pushing the complexity down to a minimal two-word paradigm offers the advantage of isolating the binding process from the contribution of other cognitive processes, such as working memory load, which may come into play for longer sentences with complex syntactic structures (see below). The study by Bemis \& Pylkkänen (2011) was one of the first studies with a minimal paradigm and inspired many other studies to use a similar design. For example, in Bemis \& Pylkkänen (2012), binding during both auditory comprehension and reading was associated with an evoked response in the 1ATL, followed by effects in the left angular gyrus. In this study (unlike in Bemis \& Pylkkänen, 2011), no effects were found in vmPFC for the two-word phrase condition compared to the wordlist condition. Pylkkänen et al. (2014) demonstrated that binding in production was associated with parallel effects in 1ATL and vmPFC. In another key study using a minimal paradigm, using fMRI Zaccarella et al. (2017) demonstrated that BA44 is involved in binding not just at the sentence level, but also at the level of three-word phrases. In all these studies using a minimal paradigm, binding occurred at both the syntactic and the semantic level. Different is the fMRI study by Zaccarella \& Friederici (2015), who used two-word phrases combining an adjective determiner with a noun. Crucially, the noun was a non-word. With this design, they investigated the neuroanatomical basis of syntactic binding in a context with minimal semantic information and found the anterior section of the ventral left pars opercularis (a subregion of BA44) to be involved.

Alongside the research on binding using a minimal paradigm, a lot of studies have been conducted on the neural basis of more complex sentence processing, introducing for example manipulations of syntactic complexity, a priming (repetition suppression) design, or a design with semantic and/or syntactic ambiguities. Mostly, these studies have revealed a left-lateralised network of regions that is associated with the processing of syntactic structures. This network includes primarily the inferior frontal and temporal cortex, and regions in the inferior parietal cortex (Friederici et al., 2003; Snijders et al., 2009; Menenti et al., 2011; Pallier et al., 2011; Papoutsi et al., 2011; e.g. den Ouden et al., 2012; Segaert et al., 2012; Tyler et al., 2013; Schoot et al., 2014; Shetreet \& Friedmann, 2014; Goucha \& Friederici, 2015; Zaccarella \& Friederici, 2015; Zaccarella et al., 2017) (for reviews see: Tyler \& Marslen-Wilson, 2008; Friederici, 2011; Hagoort \& Indefrey, 2014; Hagoort, 2017). In this line of research, some have raised the concern that manipulations of sentence complexity often go hand in hand with variations in working memory load. Indeed, some evidence suggests that activation of the left inferior frontal cortex in particular is related to syntactic working memory rather than syntactic binding (Fiebach et al., 2001, 2005). Moreover, others have suggested that the left inferior frontal gyrus is associated with general cognitive functions such as selection among different alternatives, and recovery in the case of misinterpretation (Novick et al., 2005; Rodd et al., 2010), rather than with syntactic processing per se. In sum, although some research questions clearly can only be answered using sentences with complex syntactic structures as stimuli, for studies focusing on the process of binding, a minimal sentence paradigm offers clear advantages. A minimal paradigm isolates the binding process from contributions of working memory and other general cognitive functions.

\section{Isolating syntactic binding using a minimal sentence paradigm}

In this study, we use a minimal two-word sentence paradigm to target syntactic binding processes, while minimising contributions of semantic and phonological binding as much as possible. This is unlike most previous studies using a minimal paradigm [although see Zaccarella \& Friederici (2015) for an exception], which have in common that they have manipulated binding at multiple levels at the same time. Previous studies have used real words as stimuli. When real words are placed together in a sentence or phrase, both syntactic and semantic binding take place. In this study, we follow a different approach and use pseudowords. The Dutch 'tersen' or English 'to grush' are examples of pseudoverbs. A pseudoword is a unit of text or speech that follows the orthographic and phonological rules of a language, but has no meaning in the mental lexicon. Using a minimal sentence paradigm with pseudowords, we can thus zoom in on binding at the syntactic level. Also, as we use a minimal sentence paradigm, we rule out contributions of general cognitive processes such as working memory.

We will investigate the EEG changes for the following critical comparison: a minimal sentence condition with syntactic binding (e.g. in Dutch: 'ik ters' and 'zij terst'; in English: 'I grush' and 'she grushes'), vs. a wordlist condition with no syntactic binding (e.g. in Dutch: 'cil ters' and 'cil terst'; in English: 'pob grush' and 'pob grushes'). Stimuli are presented auditorily. The second word, for example 'terst', is the target word, the onset of which we time-lock our comparison of interest to. Pseudoverbs such as 'grushes' (in English) are present in both the sentence and wordlist condition, thus in both conditions, morphological parsing occurs of stems and inflectional affixes (i.e. regular inflectional morphology). Inflectional affixes indicate the number and tense for each instance of a pseudoverb, for example 'grushes' in English is the regular third person singular.

The minimal sentence condition and wordlist condition thus differ from each other only with respect to binding taking place. In minimal sentences such as 'I grush', 'she grushes' and 'we grushed', the presence of a pronoun and the regular inflectional morphology are cues to establish a syntactic structure and for syntactic binding to occur (similar to Goucha \& Friederici, 2015). The aspects of syntactic binding we manipulate are as follows: (1) agreement: we establish agreement of number and person between the pronoun and the pseudoverb in the minimal sentence condition, but not in the wordlist condition; and (2) structure building: 'subject verb' is a sentence with a syntactic structure, while for wordlists with two verbs we cannot establish a syntactic structure. The paradigm thus allows zooming in on syntactic binding, with only a minimal contribution from semantics (i.e. the pronoun signals who the agent is). 


\section{Oscillatory changes in the EEG related to syntactic processing}

We will measure the brain's response to syntactic binding using EEG. Evoked responses in the EEG (also called event-related potentials, i.e. ERPs) are obtained by averaging event-locked EEG epochs (i.e. trials). An evoked response typically associated with syntactic manipulations is the P600 (Hagoort et al., 1993). A caveat of the event-related averaging approach is that it looks at activity that is time-locked and phase-locked to the event, while averaging out the non-phase-locked neural activity to the experimental event.

An alternative and complementary way to investigate eventrelated changes in the EEG signal is through time-frequency power changes induced in the EEG by the experimental event (i.e. the induced instead of evoked response) (see Bastiaansen et al., 2012 for a discussion of these two approaches). Although no study with a minimal paradigm has looked into the oscillatory changes associated with binding, related studies with more complex syntactic manipulations provide insight in the oscillatory changes associated with syntactic processing. Firstly, several studies have suggested that oscillatory changes in the beta range are associated with syntactic binding [see Bastiaansen \& Hagoort (2006) and Weiss \& Mueller (2012) for reviews on the role of beta in the binding of language]. Bastiaansen et al. (2010) found a progressive increase in power in the low-beta band as syntactically correct sentences unfolded. Weiss et al. (2005) found increased low-beta coherence between left-lateralised frontal and temporal regions for the processing of complex vs. simpler syntactic structures. Secondly, oscillatory changes in the theta range may play a role. Although changes in this frequency band are more commonly associated with the retrieval of items from the mental lexicon (Bastiaansen et al., 2005, 2008), Bastiaansen et al. (2010) observed in the theta band (in addition to the beta band) an increase in power as the sentence unfolded, suggesting a role for theta power in binding also. Lastly, both Bastiaansen et al. (2010) and Davidson \& Indefrey (2007) observed a reduction in alpha power following a syntactic violation, which could indicate increased syntactic processing after the violation is encountered. Some other studies have related changes in the alpha frequency band to sentence processing: one such study found an increase in alpha band power in auditory sentence processing (Krause et al., 1994) and another found increased coherence in the alpha range during sentence reading (Kujala et al., 2007). Alpha power increases have also been linked to the involvement of working memory in sentence comprehension (Meyer et al., 2013), but this would mostly play a role in working memory intensive sentence processing such as long-distance dependencies, rather than the processing of twoword sentences.

In this study, we aim to investigate binding at the syntactic level using a minimal sentence paradigm, to target the precisely timed oscillatory mechanisms associated with syntactic binding. We use two distinct but complementary analysis approaches. The first analysis approach is based on frequency bands as defined in previous literature, using a cluster-randomisation test which circumvents multiple comparisons (Maris \& Oostenveld, 2007). Guided by previous research on syntactic binding, we could expect oscillatory power changes associated with binding in the alpha, theta or beta frequency range. Given that there is some variability across the literature in what frequencies are included within a frequency band, we also use a second analysis approach that does not require us to determine pre-set frequency bands, but rather is data-driven. The second analysis approach looks at the entire range of frequencies between 3 and $30 \mathrm{~Hz}$. For this, we use the cluster-randomisation test (Maris \& Oostenveld, 2007) in a non-traditional way. Traditionally, when using the cluster-randomisation test one collapses across time, frequency or channels; a pre-defined time window, frequency range or channels of interest are based on prior literature. We here decided to, in addition to the traditional approach, not collapse across any of these dimensions. Instead, we examined which frequency, time and electrode combination shows a condition difference [similar to the approach used in some ECoG studies, e.g. Bauer et al. (2013)].

\section{The present study}

In sum, in this study, the question is how the brain's oscillatory changes relate to the syntactic binding process. To investigate the process of binding, we pushed down complexity to the basic twoword sentence level. We compare a minimal sentence condition (e.g. 'zij terst', allowing syntactic binding to occur) to a wordlist condition (e.g. 'cil terst', no syntactic binding occurs). Through the use of pseudowords, we can zoom in on binding at the syntactic level, minimising contributions from semantics. The minimal sentence condition and the wordlist condition are constructed as parallel as possible: each form of the pseudoverb is presented in both conditions (i.e. preceded by a pronoun in the minimal sentence condition, preceded by another pseudoverb in the wordlist condition). The critical comparison is for the second word, with the conditions of interest thus only differing in the extent to which the binding process can occur. Our experiment thus allowed investigating the oscillatory mechanisms through which binding of a minimal sentence happens at the syntactic level, largely excluding contributions from binding at other levels and excluding contributions of other cognitive processes such as working memory.

\section{Materials and methods}

\section{Participants}

The participants were twenty native Dutch speakers (10 male/10 female, mean age of 21 years with SD 3.1). Participants signed an informed consent that followed the guidelines of the Declaration of Helsinki. All participants were right-handed, with no visual or neurological impairments. The experiments were approved local Ethics Committee of the Social Sciences faculty of the Radboud University (Ethics Approval Number ECG2013-1308-120).

\section{Materials}

Using Wordgen (Duyck et al., 2004), we created 20 Dutch pseudoverbs: 'terzen', 'luiven', 'vekken', 'hooven', 'galden', 'gonken', 'golsen', 'zweben', 'zauwen', 'dispen', 'bogsen', 'cillen', 'dunfen', 'ziepen', 'dranen', 'bregen', 'glaven', 'nillen', 'maspen' and 'dernen'. We set Wordgen criteria to generate pseudowords with six letters, all ending in - 'en'. All pseudowords could be inflected according to regular inflectional morphology in Dutch and combined with one of six Dutch pronouns, which would yield for example 'ik ters, 'jij terst', 'hij terst', 'zij terst', 'wij terzen', 'jullie terzen' and 'zij terzen'.

These materials are inspired by Ullman et al. (1997), who created pseudoverbs in English (e.g. 'to prass' and 'to grush'). Analogous to our Dutch non-verbs, the novel English verbs that Ullman et al. created could be inflected and combined with pronouns, for example 'I grush, 'you grush', 'he grushes' and 'we grushed'.

We made audio recordings of the six pronouns and each of the 20 pseudoverbs in the 1st, 2nd and 3rd singular and plural present tense. Recordings were made with a female native speaker of Dutch. 
All created recordings were also saved as a reversed speech version, using the software program PRAAT (Boersma, 2001) which allows you to play speech in reverse. Again using PRAAT, we also created pink noise segments, which matched in length with each individual audio recording of the pronouns and non-words described above. The reversed speech and pink noise segments were used on filler trials (see below).

\section{Task and design EEG experiment}

Above described stimuli were presented to constitute a minimal sentence condition, a wordlist condition or one of three filler conditions.

The minimal sentence condition consisted of a pronoun paired with a pseudoverb in the 1st, 2nd or 3rd singular or plural present tense. The presence of the pronoun and the inflections are cues for syntactic binding to take place (i.e. establishing agreement of number and person between pronoun and pseudoverb, and building of a syntactic structure). In the wordlist condition, two pseudoverbs were presented, either of which could be in the 1st, 2nd or 3rd person. In this case, no syntactic binding between the two elements can occur. The EEG experiment consisted of 120 instances of the minimal sentence condition and 120 instances of the wordlist condition. For the EEG analyses, the analyses contrasts of interest were estimated by comparing the minimal sentence condition and the wordlist condition (i.e. a comparison of the extent to which syntactic binding occurs).

We also used the following trials as fillers. First, we included pink noise trials for variation. A pink noise trial consisted of two segments of pink noise which were matched in length with a pronoun and a verb or with two verbs (120 instances). Second, we had reversed speech trials, which were included to create a task for the participants (see below). In reversed speech trials, a segment was played in reverse (90 instances; of these, 30 contained a pronoun and a reversed pseudoverb, 30 contained a reversed pseudoverb followed by a pseudoverb, 15 contained a reversed pseudoverb and pink noise and 15 contained pink noise and a reversed pseudoverb). Lastly, there were 30 instances of a minimal sentence-mismatch condition, in which there was an agreement mistake in person and number between the pronoun and pseudoverb. These trials were inserted to ensure some continuity and similarity with the stimuli from the behavioural pre-test experiment (see below).

The participants' task was to detect reversed speech (which only occurred on filler trials). With this task, we ensured that participants paid close attention to the stimuli throughout the EEG measurements. Also, there was thus no difference in response decision processes between the crucial conditions of interest, that is the minimal sentence and the wordlist condition.

The experimental list consisted of a total of 480 trials, which were preceded by 15 practice trials to gain familiarity with the reversed speech detection task. The presentation time of each element in the trial was as follows (Fig. 1): a fixation cross was presented for $200 \mathrm{~ms}$, followed by a grey screen presented for $300 \mathrm{~ms}$, followed by a grey screen presented for $1200 \mathrm{~ms}$ of which the onset coincided with auditory presentation of the first word, followed by a grey screen presented for $1400 \mathrm{~ms}$ of which the onset coincided with auditory presentation of the second word, followed by a grey screen with two response options (yes/no) presented for $800 \mathrm{~ms}$ and followed by a grey screen presented for $1000 \mathrm{~ms}$.

\section{Behavioural pre-test experiment}

Prior to completing the EEG experiment, each participant also completed a short behavioural pre-test experiment. The pre-test experiment was conducted simply to verify that participants indeed are able to syntactically bind minimal sentences containing a pronoun and pseudoverb. This was done by including a minimal sentence condition with agreement mistakes, that is a minimal sentence-mismatch condition, and instructing participants to detect these mistakes. If participants can detect mistakes in the behavioural pre-test experiment, then we can infer from this that participants can perform syntactic binding for a pronoun with a pseudoverb.

The behavioural pre-test experiment was made up of three conditions: the minimal sentence condition (e.g. she grushes), the wordlist condition (e.g. pob grushes) and the minimal sentence-mismatch condition (e.g. I grushes). There were 60 instances of each condition. The experimental list thus consisted of a total of 180 trials, which were preceded by 45 practice trials. The presentation time of each element in the trial was identical in the behavioural pre-test and during the EEG experiment (Fig. 1).

We will list the results of the pre-test experiment here, as the aim of the pre-test experiment was to validate the manipulation of the main experiment of this study. The averaged group performance accuracy was $90.0 \%$ ( $\mathrm{SE}=1.69 \%$ ) for detecting agreement mistakes in the minimal sentence-mismatch condition, 92.9\% ( $\mathrm{SE}=1.04 \%)$ for correctly saying that there was no mistake in the minimal sentence condition, and $97.4 \%$ ( $\mathrm{SE}=2.24 \%$ ) for correctly saying that there was no mistake in the wordlist condition. Individual performance accuracy was $70 \%$ or higher. This suggests that each of the participants was able to perform the task, and thus that each individual participant who completed the EEG experiment, was able to syntactically bind a pronoun with a pseudoverb.

The results of our pre-test experiment also suggest that it is unlikely that participants are performing syntactic binding in the wordlist condition. One could be concerned that participants attempt binding in specific instances of wordlists, such as 'pob grushes' (in Dutch: 'cil terst'), if the participants were to consider 'pob' as a singular noun and 'grushes' as a pseudoverb. Note however that in the wordlist condition, the second pseudoverb was presented in the 1st, 2nd or 3rd person singular or plural. This means that we have

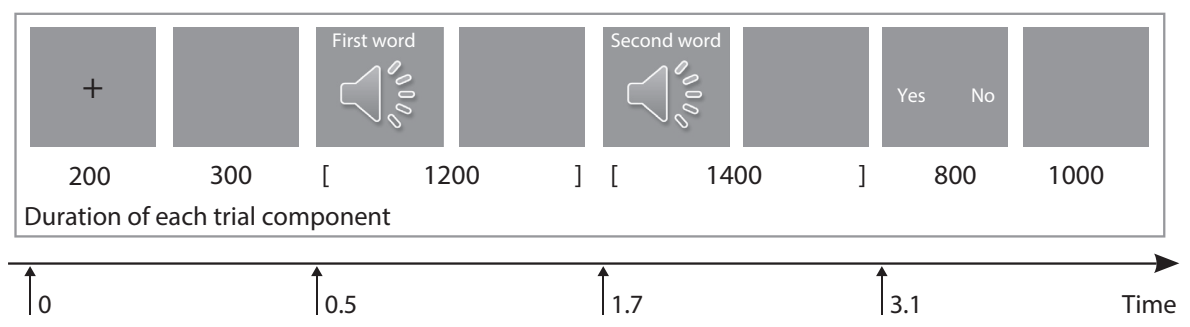

FIG. 1. Timing of each component in one trial. 
instances in the wordlist condition such as 'cil ters' ('ters' is 1st person singular, which happens in 1 of 6 cases) and 'cil tersen' ('tersen' is 1st, 2nd or 3rd person plural, which happens in 3 of 6 cases). An English equivalent would be 'pob grush'. If participants were to indeed bind these wordlists instances, they would identify $66 \%$ of the trials in the wordlist condition as having an agreement mistake in number and person between pseudonoun and pseudoverb. Given that performance accuracy was $97.4 \%$ for correctly saying that there was no mistake in the wordlist condition, it is thus unlikely that participants were performing syntactic binding in the wordlist conditions.

\section{EEG recording}

EEG recordings were made in an electromagnetically shielded room with 60 active surface electrodes placed in an equidistant montage (Acticap, Brain Products, Herrsching, Germany). An electrode on the left mastoid served as a reference; a forehead electrode served as the ground. Vertical and horizontal eye movements were recorded using electrodes on the cap in addition to an electrode placed below the left eye. Using the BrainVision Recorder Professional software (Brain Products $\mathrm{GmbH}$ ), EEG was sampled at $500 \mathrm{~Hz}$ and filtered at $0.2-200 \mathrm{~Hz}$. Impedances were kept below $20 \mathrm{k} \Omega$.

\section{Data analysis procedure}

The offline processing and analyses of the data were performed using functions from EEGLAB version 13.1.1b (Delorme \& Makeig, 2004) and the FIELDTRIP software package (Oostenveld et al., 2011), both are freely available open source Matlab toolboxes. The data were average referenced and epoched from -1.0 to $5 \mathrm{~s}$ after the onset of the visual fixation cue preceding the first word. All trials prior to being sorted into any conditions were visually inspected for non-biological signal artefacts (e.g. electrode jumps or gross movements by participants) based on visual inspection of the waveforms. Ocular and muscle artefacts were removed using independent component analysis (infomax algorithm) incorporated as the default 'runica' function in EEGLAB 13.1.1b with the first step of a PCA to reduce dimensionality of the data. We have used a similar pipeline for data analysis in previous EEG studies (e.g. van Diepen et al., 2015, 2016; van Diepen \& Mazaheri, 2017).

\section{Time-frequency representations of power}

Using the Fieldrip function 'ft_freqanalysis_mtmconv', time-frequency representations (TFRs) of power were calculated for each trial using sliding Hanning tapers having a varying time window of three cycles for each frequency $(\Delta T=3 / \mathrm{f})$, an approach which has been used in a number of previous studies (e.g. Whitmarsh et al., 2011; van Diepen et al., 2015, 2016; van Diepen \& Mazaheri, 2017). As to avoid temporal spectral leakage from previous trials, we did not baseline correct the data, but rather compared timefrequency power between the experimental conditions (Mazaheri et al., 2009).

\section{Statistical analysis}

We assessed the statistical differences in time-frequency power between the minimal sentence and wordlist condition across participants (random effects analysis) by means of the cluster-level randomisation test (incorporated in the FIELDTRIP software) proposed by Maris \& Oostenveld (2007) used in a number of previous studies (e.g. Mazaheri et al., 2009; Nieuwenhuis et al., 2009; Van Dijk et al., 2010; van Diepen et al., 2015, 2016; van Diepen \& Mazaheri, 2017). This is a conservative procedure which circumvents the type-1 error rate in a situation involving multiple comparisons (i.e. multiple channels and time-frequency points). The power of the frequencies of interest, in each channel and time point within the interval $0.5-3.1 \mathrm{~s}$ after the fixation cross (i.e. the period in between the onset of the first word and the onset of the response screen), was clustered depending on if it exceeded a dependent samples $t$-test threshold of $P<0.05$ (two-tailed). We considered a cluster to consist of at least two significant adjacent electrodes. Next, the Monte Carlo $P$ values of each cluster obtained were calculated on 1000 random partitions in which the minimal sentence and wordlist condition labels were shuffled. At each shuffle, the data were clustered again and the cluster with the largest sum of $t$-statistics entered the shuffling distribution. A $P$-value was derived by calculating the number of times the $t$-statistics in the shuffled distribution was higher than the original $t$-statistic we derived by contrasting our conditions. We considered the critical alpha level here to be 0.05 .

We followed two analysis approaches. Firstly, we followed an analysis approach in which we were guided by previous literature for the separation in pre-defined frequency bands. We performed analyses within the following frequency bands: theta $(4-7 \mathrm{~Hz})$, alpha (8-12 Hz), low-beta (15-20 Hz; Weiss \& Mueller (2012) define low-beta as $13-20 \mathrm{~Hz}$, we used $15-20 \mathrm{~Hz}$ to reduce the overlap with the tested alpha band) and high-beta $(25-30 \mathrm{~Hz}$; Weiss \& Mueller (2012) define high-beta as $20-30 \mathrm{~Hz}$, we used $25-30 \mathrm{~Hz}$ to reduce the overlap with the tested low-beta band). In these analyses, we collapsed within the frequency bands to perform cluster-level randomisation tests.

However, a caveat of collapsing across pre-defined frequency bands is that with such an approach, we are possibly overlooking changes in the oscillatory activity that are not falling within the predefined selection. As such, in a second analysis approach, rather than collapsing across frequency bands, we assessed statistical differences in power between conditions for each frequency between 3 and $30 \mathrm{~Hz}$ in $1 \mathrm{~Hz}$ increments across time and channels. As with the previous approach, power of the frequencies in each channel and time point within the interval $0.5-3.1 \mathrm{~s}$ after the fixation cross (i.e. the period in between the onset of the first word and the onset of the response screen) was clustered depending on if it exceeded a dependent samples $t$-test threshold of $P<0.05$ and the Monte Carlo $P$ values of each cluster obtained were calculated on 1000 random partitions of shuffled conditions.

These two analysis approaches are complementary, as the first is guided by previous research and the second is data-driven. To foreshadow our results, findings from both analysis approaches largely converged.

\section{Results}

\section{Behavioural results EEG experiment}

During the EEG task, we asked participants to detect reversed speech segments, to ensure they stayed attentive and listened to the stimuli. The averaged group performance accuracy was $95.5 \%$ $(\mathrm{SE}=0.65 \%)$ for detecting reversed speech during the filler trials that contained such segments and $99.6 \%(\mathrm{SE}=0.12 \%)$ for correctly answering 'No' in all other conditions. Each individual participant performed the task with high accuracy, with the worst scoring participant obtaining $88.9 \%$ accuracy for detection of the reversed speech segments. 


\section{EEG results - pre-defined frequency bands}

First, we will describe the differences in power between the minimal sentence (syntactic binding) and wordlist (no binding) conditions, revealed by analyses in pre-defined frequency bands as guided by previous literature: theta $(4-7 \mathrm{~Hz})$, alpha $(8-12 \mathrm{~Hz})$, low-beta $(15-$ $20 \mathrm{~Hz}$ ) and high-beta $(25-30 \mathrm{~Hz})$. Here, we focus first on condition differences preceding the onset of the target word (Fig. 2), then on condition differences following the onset of the target word (Fig. 3). The target word is the second word in the two-word sentence, i.e. the word for which syntactic binding occurs.

Preceding the target word, we found no significant difference in the power of theta $(4-7 \mathrm{~Hz})$ activity between the minimal sentence (syntactic binding) and the wordlist (no binding) condition. We did find a significant difference in the power of alpha $(8-12 \mathrm{~Hz})$ activity at an interval -0.4 to $0 \mathrm{~s}$ preceding the onset of the target word $(P<0.012)$ (i.e. time interval 1.3 to $1.7 \mathrm{~s}$ from the onset of fixation). Specifically, alpha power was greater in the minimal sentence (syntactic binding) condition, with the difference being most pronounced over a left temporal and central cluster of electrodes (Fig. 2A-C). We also found a significant difference in low-beta (15$20 \mathrm{~Hz})$ activity at 0.25 to $0.15 \mathrm{~s}$ before the onset of the target word $(P<0.039)$ (i.e. time interval 1.45 to $1.55 \mathrm{~s}$ from the onset of fixation). Low-beta power was significantly greater in the minimal sentence (syntactic binding) condition, most pronounced over frontal and parietal electrodes (Fig. 2D-F). These analyses revealed no effects in the high-beta $(25-30 \mathrm{~Hz})$ range.

Following the target word, we found no significant difference in the power of theta $(4-7 \mathrm{~Hz})$ activity between the minimal sentence (syntactic binding) and wordlist (no binding) condition. We did find a significant difference in alpha $(8-12 \mathrm{~Hz})$ activity at the interval 0.05 to $0.35 \mathrm{~s}$ following the target word $(P<0.035)$ (i.e. time interval 1.75 to $2.05 \mathrm{~s}$ from fixation onset). Alpha power was once again larger in the minimal sentence (syntactic binding) condition, this time most pronounced in a left-lateralised frontal-temporal cluster of electrodes. We found no power differences following the target word in low-beta $(15-20 \mathrm{~Hz})$ or high-beta $(25-30 \mathrm{~Hz})$ activity between the two conditions.

\section{EEG results - no pre-defined frequency bands}

In addition to the analyses reported above where we collapsed across pre-defined frequency bands as guided by previous literature, we took a complementary approach where we looked at differences between the minimal sentence (syntactic binding) and the wordlist (no binding) condition across every frequency between 3 and $30 \mathrm{~Hz}$ in $1 \mathrm{~Hz}$ increments. The spectrogram of Fig. 4A illustrates the number of electrodes (at a particular time and frequency) which showed a significant difference in power between the two experimental conditions.

We found that preceding the target word, significant differences between the minimal sentence (syntactic binding) and wordlist (no binding) condition were observed in a time window 0.5 to $0 \mathrm{~s}$ before the onset of the target word (i.e. a time window 1.2 to $1.7 \mathrm{~s}$ from the onset of fixation). Averaging over this time window, cluster-level randomisation tests revealed significant differences between the minimal sentence and wordlist condition in $7-14 \mathrm{~Hz}$ (i.e. alpha) power maximal over a central cluster of electrodes $(P<0.043)$ (Fig. 4B, left topoplot). Interestingly, the cluster-level randomisation test also revealed a condition difference in the $18-30 \mathrm{~Hz}$ (i.e. lowbeta and high-beta) power in a central cluster $(P<0.024)$ (Fig. 4B, middle topoplot). This suggests that the separation based on predefined frequency bands (see above; Fig. 2) can sometimes lead to overlooking possible condition effects. We should note that even though for this analysis we have averaged over a $0.5 \mathrm{~s}$ lasting time window, panel 4A shows that the beta effect is more short-lived than this.

Following the onset of the target word, significant differences between the minimal sentence (syntactic binding) and the wordlist (binding) condition were observed in the time window 0 to $0.5 \mathrm{~s}$ after the onset of the target word (i.e. a time window 1.7 to $2.2 \mathrm{~s}$ from the onset of fixation). Averaging over this time window, cluster-level randomisation tests revealed significant condition differences in $8-15 \mathrm{~Hz}$ (i.e. alpha) power, maximal over left-lateralised frontal-temporal electrodes $(P<0.028)$ (Fig. 4B, right topoplot).

In sum, the oscillatory analyses revealed the following: preceding the target word, we observed a power increase in the alpha and beta range. Following the target word, a power increase in the alpha range is associated with syntactic binding.

\section{Phase-locked activity (ERPs)}

First, we examined any differences in the P600 evoked by the presentation of words. Testing for a P600 effect in the latency range from 500 to $700 \mathrm{~ms}$ post-word, the cluster-based permutation test did not reveal any significant difference between the minimal sentence and wordlist condition in this ERP component. The evoked response for two central electrodes is illustrated in Fig. 5A.

Time-frequency representations of power in an EEG epoch capture non-phase-locked activity as well as the spectral representation of the ERP. It is therefore a possibility that the oscillatory power changes reported above (Figs 2-3) are driven by ERP effects, which are time- and phase-locked to target word onset. To investigate this possibility, we used several different approaches.

First, we used the cluster-permutation test to assess whether there were any differences in ERP amplitudes between the two conditions (Fig. 5A) in the P600 window or at any of the time intervals in which significant oscillatory differences were observed. We did not find any differences in the amplitude of the ERPs between the two conditions (i.e. no cluster of electrodes surpassed the threshold of $P<0.05)$.

Our next approach focused on the spectral representation of the ERPs in each condition. Figure 5B illustrates the difference between

FIG. 2. Differences in alpha and low-beta power between the minimal sentence and the wordlist condition preceding the onset of the target word. (A) The time course of the power envelope of $8-12 \mathrm{~Hz}$ activity (expressed in $\mu \mathrm{V}^{2}$ ) in electrodes showing the most pronounced difference between the minimal sentence (i.e. binding) vs. wordlist (i.e. no binding) condition. The grey rectangle indicates the time window in which the difference between conditions is significant $(P<0.012)$, that is 0.4 to $0 \mathrm{~s}$ prior to the onset of the target word. (B) The dots visually illustrate the cluster of electrodes that show the most pronounced condition difference for alpha power $(8-12 \mathrm{~Hz})$, over the averaged time interval in which a significant difference was found using the random-cluster-permutation test (grey rectangle in panel A). (C) The averaged time-frequency spectrum for the significant electrodes. (D) The time course of the power envelope of 15$20 \mathrm{~Hz}$ activity (expressed in $\mu \mathrm{V}^{2}$ ) in electrodes showing the most pronounced difference. The grey rectangle indicates the time window in which the difference between conditions was significant $(P<0.039)$, i.e. -0.25 to -0.15 s prior to the onset of the target word. (E) The dots visually illustrate the cluster of electrodes that show the most pronounced condition difference for low-beta $(15-20 \mathrm{~Hz})$ power, over the averaged time interval in which a significant difference was found using the random-cluster-permutation test (grey rectangle in panel D). (F) The averaged time-frequency spectrum for the significant electrodes. 

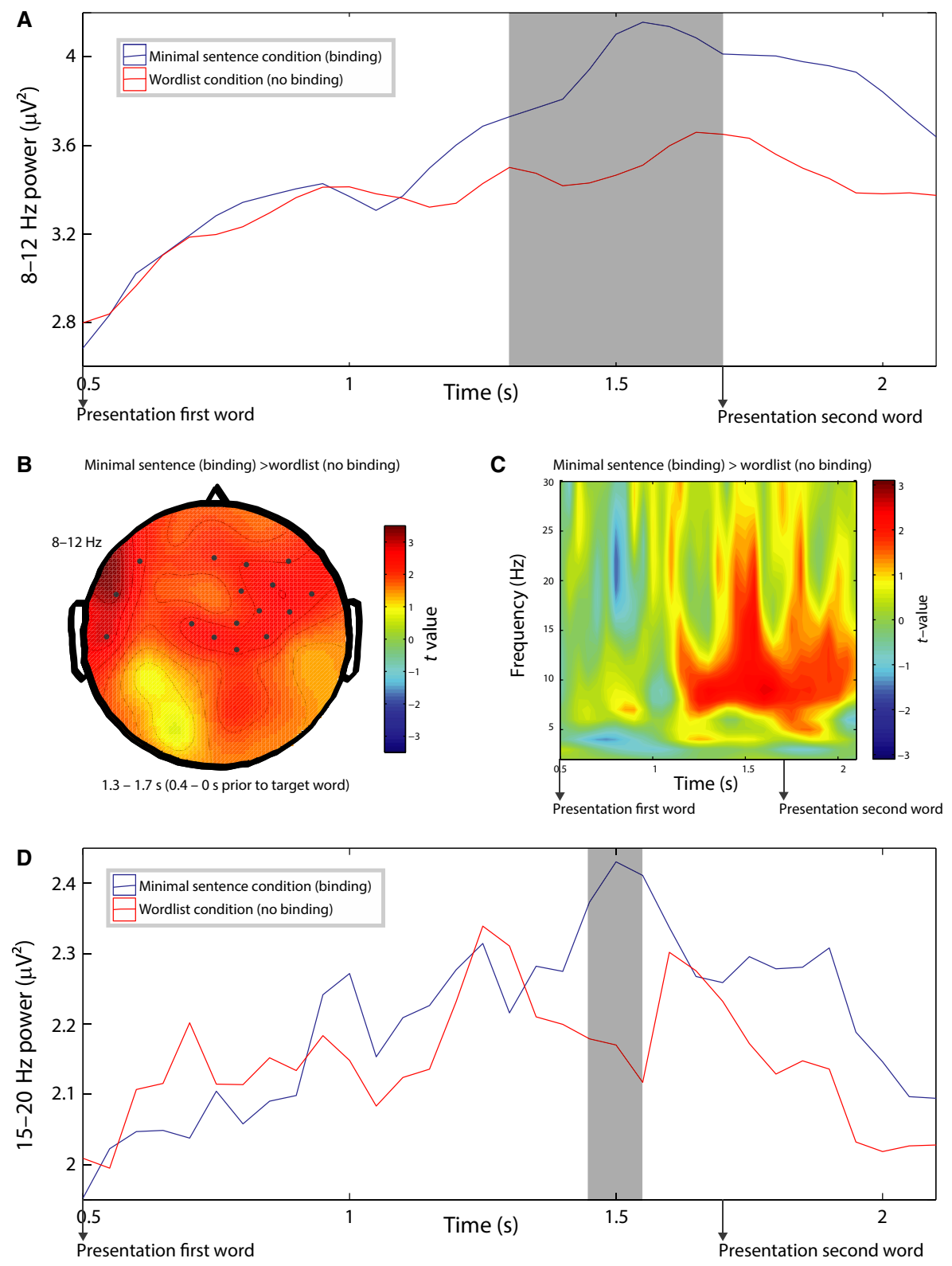

E

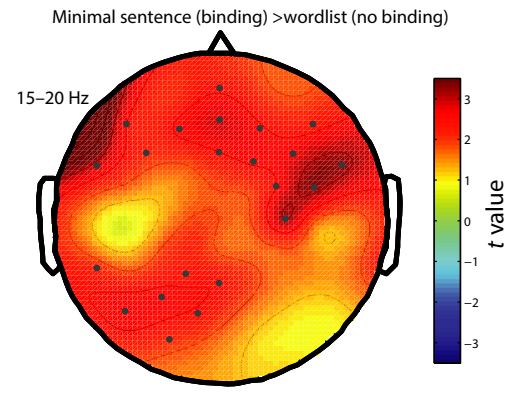

$1.45-1.55$ s $(0.25-0.15$ s prior to target word)

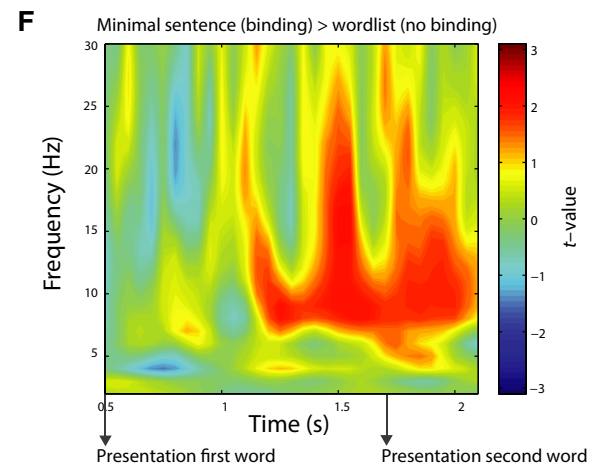

conditions and found that there were no differences in alpha (8$12 \mathrm{~Hz})$ in the time interval -0.4 to $0 \mathrm{~s}$ preceding the second word $(t(19)=1.36, P=0.18)$, in beta $(15-20 \mathrm{~Hz})$ in the time interval 0.25 to $0.15 \mathrm{~s}$ preceding the second word $(t(19)=1.30, P=0.21)$ and in alpha $(8-12 \mathrm{~Hz})$ in the time interval 0.05 to $0.35 \mathrm{~s}$ following the time-frequency representation of the ERP for the minimal sentence condition and the time-frequency representation of the ERP for the wordlist condition (i.e. the ERPs depicted in Fig. 5A). We performed $t$-tests on the time-frequency representation of these ERPs to determine whether there was a difference between the

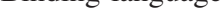




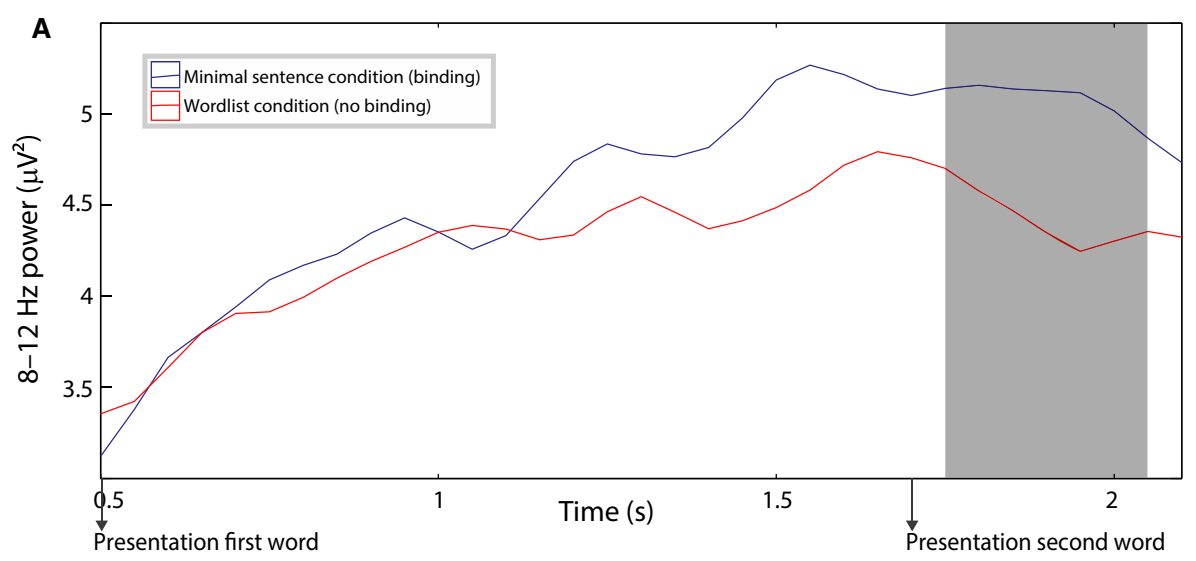

B Minimal sentence (binding) $>$ wordlist (no binding)

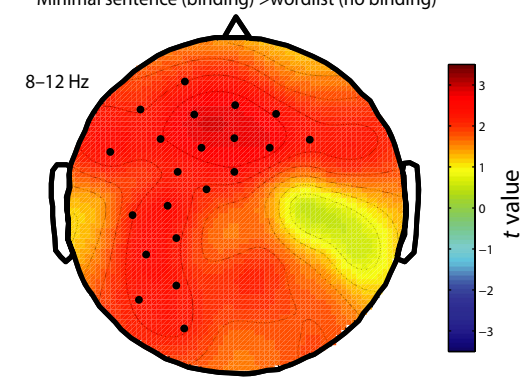

C

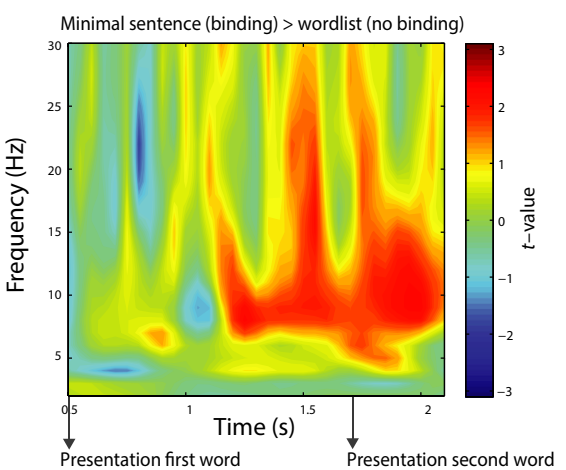

FIG. 3. Differences in alpha power between the minimal sentence and the wordlist condition following the onset of the target word. (A) The time course of the power envelope of $8-12 \mathrm{~Hz}$ activity (expressed in $\mu \mathrm{V}^{2}$ ) in electrodes showing the most pronounced difference between the minimal sentence (i.e. binding) vs. wordlist (i.e. no binding) condition. The grey rectangle indicates the time window in which the difference between conditions is significant $(P<0.035)$, that is 0.05 to $0.35 \mathrm{~s}$ following presentation of the target word. (B) The dots visually illustrate the cluster of electrodes that show the most pronounced condition difference for alpha power $(8-12 \mathrm{~Hz}$ ), over the averaged time interval in which a significant difference was found using the random-cluster-permutation test (grey rectangle in panel A). (C) The averaged time-frequency spectrum for the significant electrodes.

the target word $(t(19)=1.7, P=0.11)$. This suggests that the oscillatory differences we observed above (Figs $2-3$ ) are not likely to be driven by phase-locked activity.

Lastly, we removed the spectral components of the averaged ERP from the total spectra (i.e. phase and non-phase-locked) (Cacace \& McFarland, 2003) and reran the cluster-based permutation tests as reported above. The significance levels of our results were unchanged. Based on these analyses, it is unlikely that changes in the ERP drive the oscillatory power changes we report above.

\section{Discussion}

In this study, we investigated which oscillatory changes in brain activity elicited by the onset of words are linked to the syntactic binding process. We focused on the oscillatory changes centred around the second word (target word) in a two-word sentence, comparing the following two critical conditions: a minimal sentence for which syntactic binding occurs (e.g. in Dutch: 'ik ters' and 'zij terst', English equivalent: 'I grush' and 'she grushes') and a wordlist condition for which no syntactic binding occurs (e.g. in Dutch: 'cil ters' and 'cil terst', English equivalent: 'pob grush', 'pob grushes'). In a minimal sentence such as 'ik ters', syntactic binding involves establishing agreement of number and person between pronoun and pseudoverb, and building a syntactic structure. We followed two distinct analysis approaches (one guided by previous literature and one data-driven approach) and found a largely converging pattern of results. The results can be described as follows. Preceding the onset of the target word, in the condition where the target word is to be bound together with the preceding word in the two-word sentence, there was a power increase in the alpha frequency band $(8-12 \mathrm{~Hz})$ and low-beta frequency band $(15-20 \mathrm{~Hz})$, respectively $0.4-0 \mathrm{~s}$ before and 0.25 to $0.15 \mathrm{~s}$ before the onset of the target word. Our second analysis confirmed this and revealed that also in the highbeta band, there was a significant condition difference preceding the target word (i.e. effects observed 7-14 and 18-30 Hz). The effects preceding the target word were observed mostly in central electrodes. Following the onset of the target, there was a power increase in left frontal-temporal electrodes in the alpha frequency band (8$12 \mathrm{~Hz}$ ) for the minimal sentence compared to the wordlist condition, in a time window 0.05 to $0.35 \mathrm{~s}$ following presentation of the target word, indicative of the process of binding taking place. Converging findings were observed based on our second analysis approach, that is a condition difference from 8 to $15 \mathrm{~Hz}$ following the target word.

We believe that these findings could be interpreted against the backdrop of theoretical frameworks proposing that a left-lateralised network of frontal-temporal areas is associated with syntactic binding (e.g. Hagoort, 2003, 2009; Tyler \& Marslen-Wilson, 2008). Hagoort $(2003,2005,2009)$ argues that the left inferior frontal gyrus binds information while the left posterior temporal cortex is responsible for the retrieval of the materials that are needed (e.g. information about syntactic categories, number and gender). Both aspects played a role in our design: for example for 'ters', information needs to be retrieved to determine that this is the inflection for a first person singular; then, the pseudoverb 'ters' is syntactically bound to 


\section{A Minimal sentence (binding) > wordlist (no binding)}

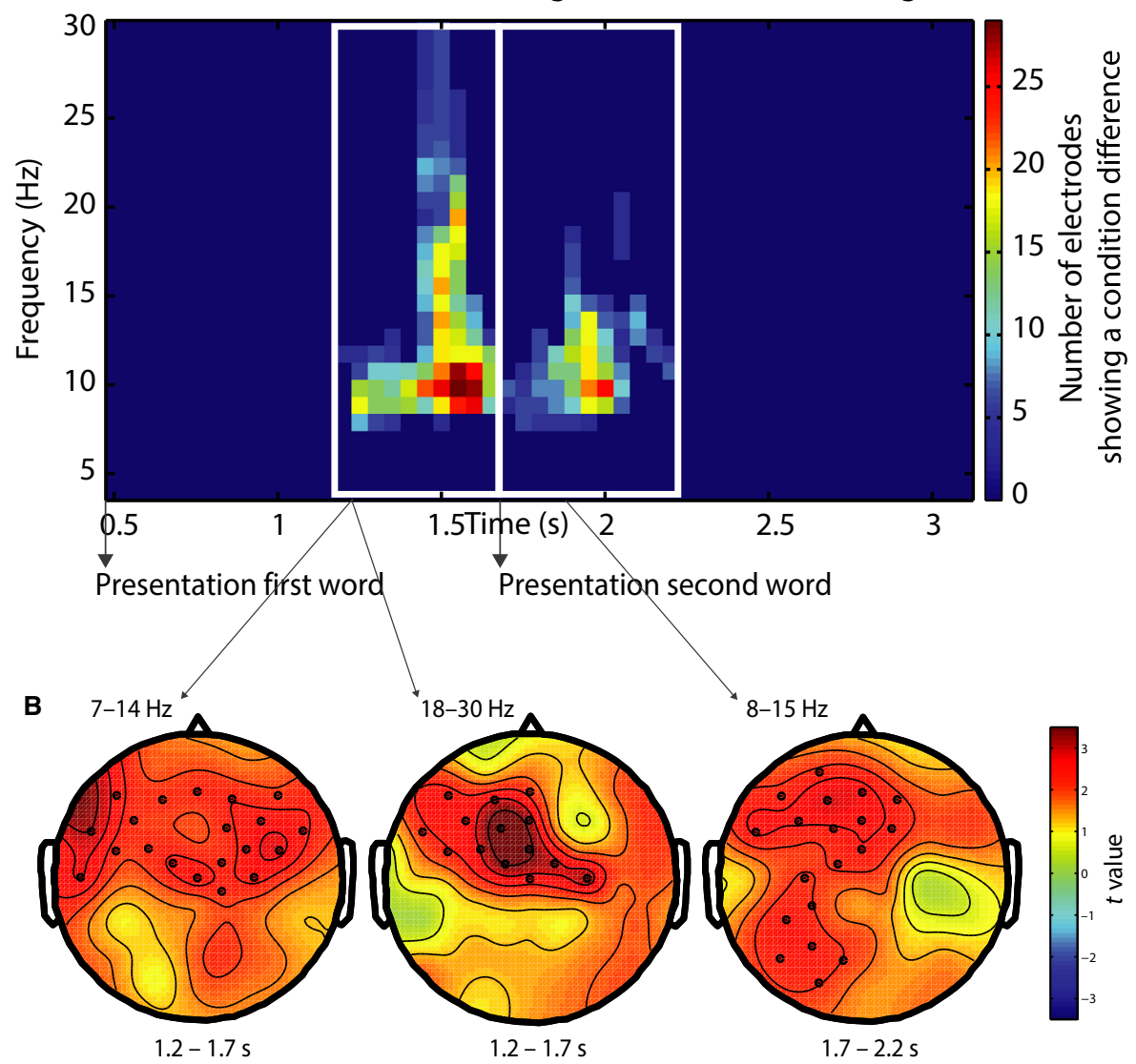

FIG. 4. A data-driven approach to assessing the differences in power between the minimal sentence and the wordlist condition. (A) Illustrated is the number of electrodes for which a significant difference in power between the minimal sentence (syntactic binding) and the wordlist (no binding) condition was observed, for each frequency and timepoint combination. The values in this graph range from 0 to 26 , with the numerical value, thus indicating the number of electrodes for which a condition difference was observed for a specific frequency/timepoint. We masked activity that was significant in less than three electrodes (value set back to 0). This figure clearly illustrates that preceding the target word, condition differences are observed in the time window from 1.2 to $1.7 \mathrm{~s}$ (left white rectangle). Following the target word, condition differences are observed in the time window from 1.7 to $2.2 \mathrm{~s}$ (right white rectangle). (B) Averaging over the time window from 1.2 to $1.7 \mathrm{~s}$, significant differences between the minimal sentence and wordlist condition are observed for $7-14 \mathrm{~Hz}$ power (condition difference maximal over a central cluster - leftmost topoplot) and for $18-30 \mathrm{~Hz}$ power (condition difference maximal over a central cluster - middle topoplot). Averaging over the time window from 1.7 to $2.2 \mathrm{~s}$, significant differences between the minimal sentence and wordlist condition are observed for $8-15 \mathrm{~Hz}$ power, most pronounced over left-lateralised frontal and temporal electrodes (rightmost topoplot). The dots represent electrodes that showed a significant difference $(P<0.05)$

the preceding pronoun 'ik'. For target words that could be bound to the preceding pronoun in the minimal two-word sentence (compared to the wordlist condition), we found a power increase in left-lateralised frontal-temporal electrodes in the alpha frequency band (8$12 \mathrm{~Hz}$ ) following the onset of the target word.

We also found increases in power in the alpha, low-beta and high-beta frequency bands immediately preceding the onset of the second word. This could be interpreted as neural responses for the expectation of binding needing to occur. In our paradigm, participants can expect that binding will need to occur when the first word of the two-word sentence is a pronoun. Likewise, when the first word is a pseudoverb, participants can expect that no binding will need to happen. This is analogous to how syntactic binding would occur in natural language processing. When comprehending sentences, words come in one at a time. We expect the sentence to unfold further and can anticipate that upcoming words will need to be bound to the words that are already presented to us. Bastiaansen et al. (2010) observed a progressive increase in power in the theta and low-beta band as the sentence unfolded. Importantly, both Wang et al. (2017) and Rommers et al. (2017) report evidence for the role of alpha power modulations in predicting upcoming language input. Moreover, beta oscillations have been shown to have an important role in top-down predictions, stimulus expectation and maintenance of information (Weiss \& Mueller, 2012; Lewis \& Bastiaansen, 2015; Lewis et al., 2015, 2016). Thus, we seem to have observed a signature of an expectation for the sentence to unfold further and binding needing to take place.

The oscillatory mechanisms that emerged in our study are only partly in line with findings of previous studies on syntactic processing in sentences with more complex structures. A common finding in previous literature is (following the target word) an oscillatory change in the beta range associated with syntactic binding (Weiss et al., 2005; Bastiaansen \& Hagoort, 2006; Bastiaansen et al., 2010; Weiss \& Mueller, 2012). We find an increase in the beta frequency range, not following but directly preceding the word for which binding occurs, which, as discussed above, may be a neural signature for the expectation of binding needing to occur.

Following the target word, we find an increase in the alpha frequency range associated with syntactic binding. The cluster of electrodes has a left-lateralised frontal-temporal topography, which 

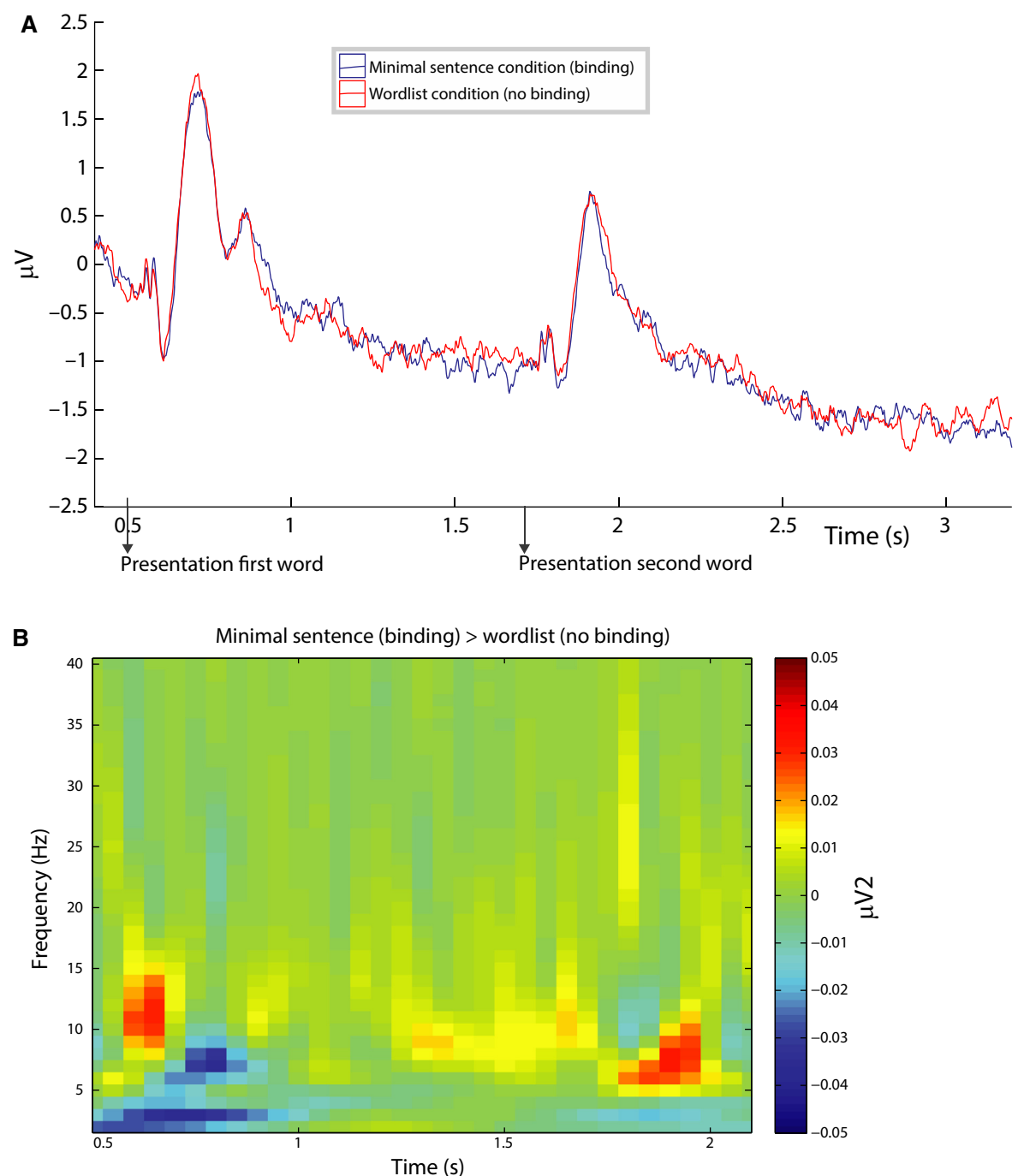

FIG. 5. Phase-locked activity. (A) The evoked response is illustrated for two central electrodes (corresponding to electrodes 1 and 2 on the equidistant electrode montage as displayed in figure 1 of Simanova et al. (2010)), for the minimal sentence and the wordlist condition. (B) The difference between the timefrequency representation of the ERP for the minimal sentence condition and the time-frequency representation of the ERP for the wordlist condition.

is in line with several theoretical proposals on binding at the syntactic level (e.g. Hagoort, 2003, 2009; Tyler \& Marslen-Wilson, 2008). Weiss \& Mueller (2012) proposed a role for beta oscillations in binding, but our findings suggest that this may also extend into the alpha frequency range. Our finding could be in line with a previous study showing an alpha power increase for processing auditory sentences (Krause et al., 1994; Meyer et al., 2013). However, two other previous studies (Davidson \& Indefrey, 2007; Bastiaansen et al., 2010) demonstrated that syntactic violations were followed by a reduction in alpha power, suggesting that an alpha suppression effect is associated with increased syntactic processing after the violation is encountered. We however find an increase (not a decrease) in alpha power related to syntactic binding. Murphy (2015) proposed the alpha and beta band to be crucial for the linguistic computations concatenation and labelling, and thus, the present findings could be in line with his model. Our finding cannot readily be reconciled with the commonly attributed role of alpha oscillations to accessing a knowledge system (Klimesch, 2012), as according to this proposal, task-relevant regions would show an alpha suppression effect while task-irrelevant regions would show an increase in alpha power. Our findings could thus suggest that we may be able to attribute an additional mechanism to oscillatory changes in the alpha band, but more research is needed to investigate this further.

It must be noted that unlike previous studies investigating oscillatory changes related to syntactic processing, we used a minimal sentence paradigm with pseudoverbs. Such a paradigm offers several advantages. Firstly, with this approach, we zoomed in on binding at the syntactic level, with minimal contributions from semantics and phonology. All oscillatory signatures we find can therefore directly be related to the process of syntactic binding. Second, we studied binding at the most basic level and pushed the complexity down to a minimal two-word sentence. There is likely little contribution from general cognitive functions such as working memory (a concern that has been raised for previous studies on syntactic processing using more complex syntactic structures). Processing phrases or sentences with a length of two words involves minimal cognitive load (Zaccarella \& Friederici, 2015). Future research will have to determine whether our paradigm choice can explain some of the divergence between our findings and the findings of studies investigating oscillatory changes associated with more complex sentence processing. 
Though with our paradigm we have zoomed in on syntactic binding, we believe that neither our results nor our interpretation of the results necessarily needs to be specific for syntactic binding only, and not even for the binding of linguistic elements. It may well be that in future studies, similar oscillatory mechanisms are observed for semantic, phonological or other forms of binding.

Lastly, we have used two complementary analysis approaches and have showed that both led to a largely convergent pattern of findings. In our second analysis approach, we were not led by specific frequency bands as defined previously in the literature, but rather looked at each frequency between 3 and $30 \mathrm{~Hz}$ in $1 \mathrm{~Hz}$ increments. We uncovered effects in the high-beta range in our second analyses approach, suggesting that a separation based on pre-defined frequency bands could in some cases lead to overlooking condition differences. With this, our paper offers a methodological advance which may be particularly useful in future investigations where researchers do not have a priori hypotheses about particular frequency bands involved in a task. This may prove particularly useful in a field like psycholinguistics, for which (in comparison with other cognitive domains) relatively little previous literature on neural oscillations is available.

In sum, we investigated the oscillatory mechanisms through which syntactic binding occurs in a minimal sentence paradigm. In the syntactic binding condition, a power increase was observed in the alpha and beta frequency range shortly preceding the presentation of a word that requires binding (relative to when the word is not bound with the preceding linguistic context). These signatures may relate to language comprehenders expecting the need for binding to occur. Following the presentation of the target word in a binding context, an increase in alpha power is observed in a leftlateralised cluster of frontal-temporal electrodes (a brain network known to be involved in binding). This alpha increase could be a neural signature for binding taking place.

\section{Acknowledgements}

The authors thank Laura Arendsen, who created the audio recordings of the stimuli and thank Charlotte Poulisse and Laura Arendsen for assistance in collection of the EEG data.

\section{Conflict of interest}

There is no conflict of interests.

\section{Author contributions}

KS and PH designed the study. KS collected the data. KS and AM analysed the data. KS wrote the manuscript. KS, AM and PH edited the manuscript.

\section{Data accessibility}

All data and processing scripts are available upon email request to corresponding author.

\section{References}

Bastiaansen, M. \& Hagoort, P. (2006) Oscillatory neuronal dynamics during language comprehension. Prog. Brain Res., 159, 179-196.

Bastiaansen, M., Van Der Linden, M., Ter Keurs, M., Dijkstra, T. \& Hagoort, P. (2005) Theta responses are involved in lexical-semantic retrieval during language processing. J. Cognitive Neurosci., 17, 530-541.
Bastiaansen, M., Oostenveld, R., Jensen, O. \& Hagoort, P. (2008) I see what you mean: theta power increases are involved in the retrieval of lexical semantic information. Brain Lang., 106, 15-28.

Bastiaansen, M., Magyari, L. \& Hagoort, P. (2010) Syntactic unification operations are reflected in oscillatory dynamics during on-line sentence comprehension. J. Cognitive Neurosci., 22, 1333-1347.

Bastiaansen, M., Mazaheri, A. \& Jensen, O. (2012). Beyond ERPs: oscillatory neuronal dynamics. In Luck, S. J., \& Kappenman, E. S. (Eds), The Oxford Handbook of Event-Related Potential Components. Oxford University Press, New York, pp. 31-50.

Bauer, P.R., Vansteensel, M.J., Bleichner, M.G., Hermes, D., Ferrier, C.H., Aarnoutse, E.J. \& Ramsey, N.F. (2013) Mismatch between electrocortical stimulation and electrocorticography frequency mapping of language. Brain Stimul., 6, 524-531

Bemis, D.K. \& Pylkkänen, L. (2011) Simple composition: a magnetoencephalography investigation into the comprehension of minimal linguistic phrases. J. Neurosci., 31, 2801-2814.

Bemis, D.K. \& Pylkkänen, L. (2012) Basic linguistic composition recruits the left anterior temporal lobe and left angular gyrus during both listening and reading. Cereb. Cortex, 23, 1859-1873.

Boersma, P. (2001) Praat, a system for doing phonetics by computer. Glot Int., 5: 9/10, 341-345.

Cacace, A.T. \& McFarland, D.J. (2003) Spectral dynamics of electroencephalographic activity during auditory information processing. Hearing Res., 176, 25-41.

Chomsky, N. (1995). The Minimalist Program. The MIT Press, Cambridge, Massachussets.

Davidson, D.J. \& Indefrey, P. (2007) An inverse relation between eventrelated and time-frequency violation responses in sentence processing. Brain Res., 1158, 81-92.

Delorme, A. \& Makeig, S. (2004) EEGLAB: an open source toolbox for analysis of single-trial EEG dynamics including independent component analysis. J. Neurosci. Meth., 134, 9-21.

van Diepen, R.M. \& Mazaheri, A. (2017) Cross-sensory modulation of alpha oscillatory activity: suppression, idling and default resource allocation. Eur. J. Neurosci., 45, 1431-1438.

van Diepen, R.M., Cohen, M.X., Denys, D. \& Mazaheri, A. (2015) Attention and temporal expectations modulate power, not phase, of ongoing alpha oscillations. J. Cognitive Neurosci., 27, 1573-1586.

van Diepen, R.M., Miller, L.M., Mazaheri, A. \& Geng, J.J. (2016) The role of alpha activity in spatial and feature-based attention. eNeuro, 3, ENEURO. 0204-0216.2016.

Duyck, W., Desmet, T., Verbeke, L.P.C. \& Brysbaert, M. (2004) WordGen: a tool for word selection and nonword generation in Dutch, English, German, and French. Behav. Res. Meth. Ins. C., 36, 488-499.

Fiebach, C.J., Schlesewsky, M. \& Friederici, A.D. (2001) Syntactic working memory and the establishment of filler-gap dependencies: insights from ERPs and fMRI. J. Psycholinguist. Res., 30, 321-338.

Fiebach, C.J., Schlesewsky, M., Lohmann, G., von Cramon, D.Y. \& Friederici, A.D. (2005) Revisiting the role of Broca's area in sentence processing: syntactic integration versus syntactic working memory. Hum. Brain Mapp., 24, 79-91.

Friederici, A.D. (2011) The brain basis of language processing: from structure to function. Physiol. Rev., 91, 1357-1392.

Friederici, A.D., Rueschemeyer, S.-A., Hahne, A. \& Fiebach, C.J. (2003) The role of left inferior frontal and superior temporal cortex in sentence comprehension: localizing syntactic and semantic processes. Cereb. Cortex, 13, 170-177.

Goucha, T. \& Friederici, A.D. (2015) The language skeleton after dissecting meaning: a functional segregation within Broca's Area. NeuroImage, 114, 294-302.

Grodzinsky, Y. \& Friederici, A.D. (2006) Neuroimaging of syntax and syntactic processing. Curr. Opin. Neurobiol., 16, 240-246.

Hagoort, P. (2003) How the brain solves the binding problem for language: a neurocomputational model of syntactic processing. Neurolmage, 20, S18-S29.

Hagoort, P. (2005) On Broca, brain, and binding: a new framework. Trends Cogn. Sci., 9, 416-423.

Hagoort, P. (2009). Reflections on the neurobiology of syntax. In Bickerton, D. \& Szathmáry, E. (Eds), Biological Foundations and Origin of Syntax. MIT Press, Cambridge, MA.

Hagoort, P. (2016). A model on the neurobiology of language beyond single word processing. In Hickok, G. \& Small, S. (Eds), Neurobiology of Language. Elsevier, Amsterdam, pp. 339-347. 
Hagoort, P. (2017) The core and beyond in the language-ready brain. Neurosci. Biobehav. Rev., 81, 194-204.

Hagoort, P. \& Indefrey, P. (2014) The neurobiology of language beyond single words. Annu. Rev. Neurosci., 37, 347-362.

Hagoort, P., Brown, C. \& Groothusen, J. (1993) The syntactic positive shift (SPS) as an ERP measure of syntactic processing. Lang. Cogn. Process., 8, 439-483.

Klimesch, W. (2012) Alpha-band oscillations, attention, and controlled access to stored information. Trends Cogn. Sci., 16, 606-617.

Krause, C.M., Lang, H.A., Laine, M., Helle, S.I., Kuusisto, M.J. \& Pörn, B. (1994) Event-related desynchronization evoked by auditory stimuli. Brain Topogr., 7, 107-112.

Kujala, J., Pammer, K., Cornelissen, P., Roebroeck, A., Formisano, E. \& Salmelin, R. (2007) Phase coupling in a cerebro-cerebellar network at 8$13 \mathrm{~Hz}$ during reading. Cereb. Cortex, 17, 1476-1485.

Lewis, A.G. \& Bastiaansen, M. (2015) A predictive coding framework for rapid neural dynamics during sentence-level language comprehension. Cortex, 68, 155-168.

Lewis, A.G., Wang, L. \& Bastiaansen, M. (2015) Fast oscillatory dynamics during language comprehension: unification versus maintenance and prediction? Brain Lang., 148, 51-63.

Lewis, A.G., Schoffelen, J.-M., Schriefers, H. \& Bastiaansen, M. (2016) A predictive coding perspective on beta oscillations during sentence-level language comprehension. Front. Hum. Neurosci., 10, 85.

Maris, E. \& Oostenveld, R. (2007) Nonparametric statistical testing of EEGand MEGdata. J. Neurosci. method., 164, 177-190.

Mazaheri, A., Nieuwenhuis, I.L., van Dijk, H. \& Jensen, O. (2009) Prestimulus alpha and mu activity predicts failure to inhibit motor responses. Hum. Brain Mapp., 30, 1791-1800.

Menenti, L., Gierhan, S.M.E., Segaert, K. \& Hagoort, P. (2011) Shared language: overlap and segregation of the neuronal infrastructure for speaking and listening revealed by functional MRI. Psychol. Sci., 22, 1173-1182.

Meyer, L., Obleser, J. \& Friederici, A.D. (2013) Left parietal alpha enhancement during working memory-intensive sentence processing. Cortex, 49, 711-721.

Murphy, E. (2015) The brain dynamics of linguistic computation. Front. Psychol., 6, 1515.

Nieuwenhuis, I., Takashima, A., Oostenveld, R., Fernández, G. \& Jensen, O. (2009) Memory consolidation increases the involvement of and the connectivity between neocortical memory areas; an MEG study. NeuroImage, 47, S76.

Novick, J.M., Trueswell, J.C. \& Thompson-Schill, S.L. (2005) Cognitive control and parsing: reexamining the role of Broca's area in sentence comprehension. Cogn. Affect. Behav. Ne., 5, 263-281.

Oostenveld, R., Fries, P., Maris, E. \& Schoffelen, J.-M. (2011) FieldTrip: open source software for advanced analysis of MEG, EEG, and invasive electrophysiological data. Comput. Intel. Neurosc., 2011, 156869.

den Ouden, D.-B., Saur, D., Mader, W., Schelter, B., Lukic, S., Wali, E., Timmer, J. \& Thompson, C.K. (2012) Network modulation during complex syntactic processing. NeuroImage, 59, 815-823.

Pallier, C., Devauchelle, A.-D. \& Dehaene, S. (2011) Cortical representation of the constituent structure of sentences. Proc. Natl. Acad. Sci., 108, 2522-2527.

Papoutsi, M., Stamatakis, E.A., Griffiths, J., Marslen-Wilson, W.D. \& Tyler, L.K. (2011) Is left fronto-temporal connectivity essential for syntax? Effective connectivity, tractography and performance in left-hemisphere damaged patients. NeuroImage, 58, 656-664.
Pylkkänen, L., Bemis, D.K. \& Elorrieta, E.B. (2014) Building phrases in language production: an MEG study of simple composition. Cognition, 133, 371-384.

Rodd, J.M., Longe, O.A., Randall, B. \& Tyler, L.K. (2010) The functional organisation of the fronto-temporal language system: evidence from syntactic and semantic ambiguity. Neuropsychologia, 48, 1324-1335.

Rommers, J., Dickson, D.S., Norton, J.J., Wlotko, E.W. \& Federmeier, K.D. (2017) Alpha and theta band dynamics related to sentential constraint and word expectancy. Lang. Cogn. Neurosci., 32, 576-589.

Schoot, L., Menenti, L., Hagoort, P. \& Segaert, K. (2014) A little more conversation - the influence of communicative context on syntactic priming in brain and behavior. Front. Psychol., 4, 208.

Segaert, K., Menenti, L., Weber, K., Petersson, K.M. \& Hagoort, P. (2012) Shared syntax in language production and language comprehension-An fMRI study. Cereb. Cortex, 22, 1662-1670.

Shetreet, E. \& Friedmann, N. (2014) The processing of different syntactic structures: fMRI investigation of the linguistic distinction between whmovement and verb movement. J. Neurolinguist., 27, 1-17.

Simanova, I., Van Gerven, M., Oostenveld, R. \& Hagoort, P. (2010) Identifying object categories from event-related EEG: toward decoding of conceptual representations. PLOS ONE, 5, e14465.

Snijders, T.M., Vosse, T., Kempen, G., Van Berkum, J.J.A., Petersson, K.M. \& Hagoort, P. (2009) Retrieval and unification of syntactic structure in sentence comprehension: an fMRI study using word-category ambiguity. Cereb. Cortex, 19, 1493-1503.

Tyler, L.K. \& Marslen-Wilson, W. (2008) Fronto-temporal brain systems supporting spoken language comprehension. Philos. T. Roy. Soc. B, 363, 1037-1054.

Tyler, L.K., Cheung, T.P., Devereux, B.J. \& Clarke, A. (2013) Syntactic computations in the language network: characterizing dynamic network properties using representational similarity analysis. Front. Psychol., 4, 271.

Ullman, M.T., Corkin, S., Coppola, M., Hickok, G., Growdon, J.H., Koroshetz, W.J. \& Pinker, S. (1997) A neural dissociation within language: evidence that the mental dictionary is part of declarative memory, and that grammatical rules are processed by the procedural system. J. Cognitive Neurosci., 9, 266-276.

Van Dijk, H., Nieuwenhuis, I.L. \& Jensen, O. (2010) Left temporal alpha band activity increases during working memory retention of pitches. Eur. J. Neurosci., 31, 1701-1707.

Wang, L., Hagoort, P. \& Jensen, O. (2017) Language prediction is reflected by coupling between frontal gamma and posterior alpha oscillations. $J$. Cognitive Neurosci., 30, 432-447.

Weiss, S. \& Mueller, H.M. (2012) "Too many betas do not spoil the broth": the role of beta brain oscillations in language processing. Front. Psychol., 3, 201.

Weiss, S., Mueller, H.M., Schack, B., King, J.W., Kutas, M. \& Rappelsberger, P. (2005) Increased neuronal communication accompanying sentence comprehension. Int. J. Psychophysiol., 57, 129-141.

Whitmarsh, S., Nieuwenhuis, I.L., Barendregt, H. \& Jensen, O. (2011) Sensorimotor alpha activity is modulated in response to the observation of pain in others. Front. Hum. Neurosci., 5, 91.

Zaccarella, E. \& Friederici, A.D. (2015) Merge in the human brain: a subregion based functional investigation in the left pars opercularis. Front. Psychol., 6, 1818.

Zaccarella, E., Meyer, L., Makuuchi, M. \& Friederici, A.D. (2017) Building by syntax: the neural basis of minimal linguistic structures. Cereb. Cortex, 27, 411-421. 